\title{
Liver Mass as a First Presentation of Missed Malignant Melanoma
}

Imad Ghazzawi, Nazmi Kamal and Ra'ed Smadi*

KHMC, Royal Medical Services of Jordan, Jordan

\begin{abstract}
Here we present a case of multiple systemic metastasis of missed malignant melanoma in a 26 year old male who was admitted to medical floor because of abdominal pain and B- symptoms. Abdominal Ultrasound revealed Right liver lobe mass with hepatomegaly.
\end{abstract}

True cut biopsy of liver mass revealed an undifferntiated malignant tumor. The tumor cells were immunoreactive for Melan-A, Vimentin and focally for S-100 protein where the diagnosis of metastatic malignant melanoma was made.

\section{Introduction}

Malignant melanoma is a neoplasm of melanocytes with annual incidence that increased dramatically over the past few decades. Melanomas have 2 growth phases, radial and vertical. The majority of melanomas are in the skin, but other sites include the eyes, mucosa, gastrointestinal tract, genitourinary tract, and leptomeninges. Metastatic melanoma with an unknown primary site may be found in lymph nodes only.

Early presentation surgical excision is curative but late presentation associated with increased incidence of mortality.

\section{Case presentation}

Our patient is a 26 year old gentle man who was admitted to our hospital complaining of easily fatigability with abdominal pain which associated with significant loss of weight and loss of appetite for the last 4 months. A full history and physical examination was performed which showed: pale sweaty patient with abdominal distention, exophthalmos, multiple scalp nodules, multiple subcutaneous nodules, axillary and inguinal Lymphadenopathy and scar in right gluteal area where patient stated that a "lesion" was removed from that area at a private clinic which was not submitted for histopathological diagnosis.

Complete blood count showed mild anemia with a hemoglobin of $12 \mathrm{~g} / \mathrm{dl}$. Liver function tests showed very high lactate dehydrogenase (LDH) of $2472 \mathrm{IU} / \mathrm{L}$.

Abdominal ultra sound showed hepatomegaly with right liver lobe mass and thrombosed inferior vena cava. True cut biopsy of liver mass revealed an undifferntiated malignant tumor. The tumor cells were immunoreactive for Melan-A, Vimentin and focally for S-100 protein Staging work up showed the following: Brain MRI: enhancing lesion in left orbital cavity causing compression of optical nerve to left with small enhancing occipital lesion. In addition to the multiple soft subcutaneous scalp nodules.

Chest and abdomen CT scan multiple infiltrative lung nodules, and multiple peritoneal nodules were seen. Two skin biopsies from scalp and abdominal wall showed a similar metastatic tumor morphologically and immunohistochemically to that present in the liver.

\section{Discussion}

Malignant melanoma incidence and overall mortality rate are rising in recent decades. Surgery is the definitive treatment for earlystage melanoma, with medical management generally reserved for adjuvant treatment of advanced melanoma.

Melanomas may develop in or near a previously existing precursor lesion or in healthy-appearing skin. Many of these melanomas are induced by solar irradiation. Melanoma also may occur in unexposed areas of the skin, including the palms, soles, and perineum.

Early presentation and accurate diagnosis is curative while late presentation as in our patient is unfortunately is associated with increased risk of mortality and decreased survival rate.

Most of recent essential of melanoma stressed on early diagnosis as the disease is curative where this is achieved by patient attention to abnormal moles or pigmented lesion. Unfortunately the incriminated lesion in our patient is not seen only after causing symptoms after irritation by belt (Figure 1). The recognition of malignant lesion by general physicians is underestimated and passed undiagnosed and this is what happened with our patient where the lesion is excised by electrocuatery and discard with no histopathology reading.

The thorough history and thorough physical examination by resident or hospital staff is essential to add the differential diagnosis of suspected liver mass.

The dermatology consultation which aid in diagnosis of this case is very important as team work is always helpful in diagnosis of complicated difficult cases.

The staging work up showed very high LDH which indicate the disease activity, also brain MRI has right orbital metastasis and occipital metastasis.

Chest, abdomen, and pelvic CT scans showed multiple lung, liver, and rib metastasis with thrombosed inferior vena cava and multiple retroperitoneal lymphadenopathy (Figure 2).

Also multiple skin metastases mainly on anterior abdominal wall and scalp (Figure 3). We presume that the primary site of the tumor is the gluteal area where a "lesion" was excised and never submitted for histopathological examination.

Unfortunately with this advanced distant metastatic malignant melanoma the median survival is short and the available treatment

\footnotetext{
* Corresponding author: Ra'ed Smadi, KHMC, Royal Medical Services of Jordan, Jordan, Tel: 962777417252; E-mail: smadiraed@yahoo.com

Received September 15, 2014; Accepted November 17, 2014; Published November 20, 2014

Citation: Ghazzawi I, Kamal N, Smadi R (2014) Liver Mass as a First Presentation of Missed Malignant Melanoma. Pigmentary Disorders 2:152. doi:10.4172/23760427.1000152

Copyright: ( 2014 Ghazzawi I et al. This is an open-access article distributed under the terms of the Creative Commons Attribution License, which permits unrestricted use, distribution, and reproduction in any medium, provided the original author and source are credited.
} 
Citation: Ghazzawi I, Kamal N, Smadi R (2014) Liver Mass as a First Presentation of Missed Malignant Melanoma. Pigmentary Disorders 2:152. doi:10.4172/2376-0427.1000152



Figure 1: Incriminated lesion

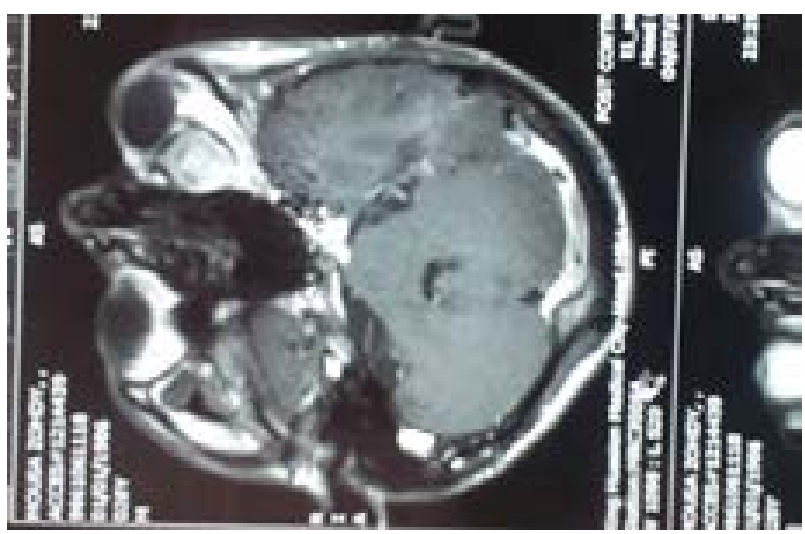

Figure 2: CT Scan Results

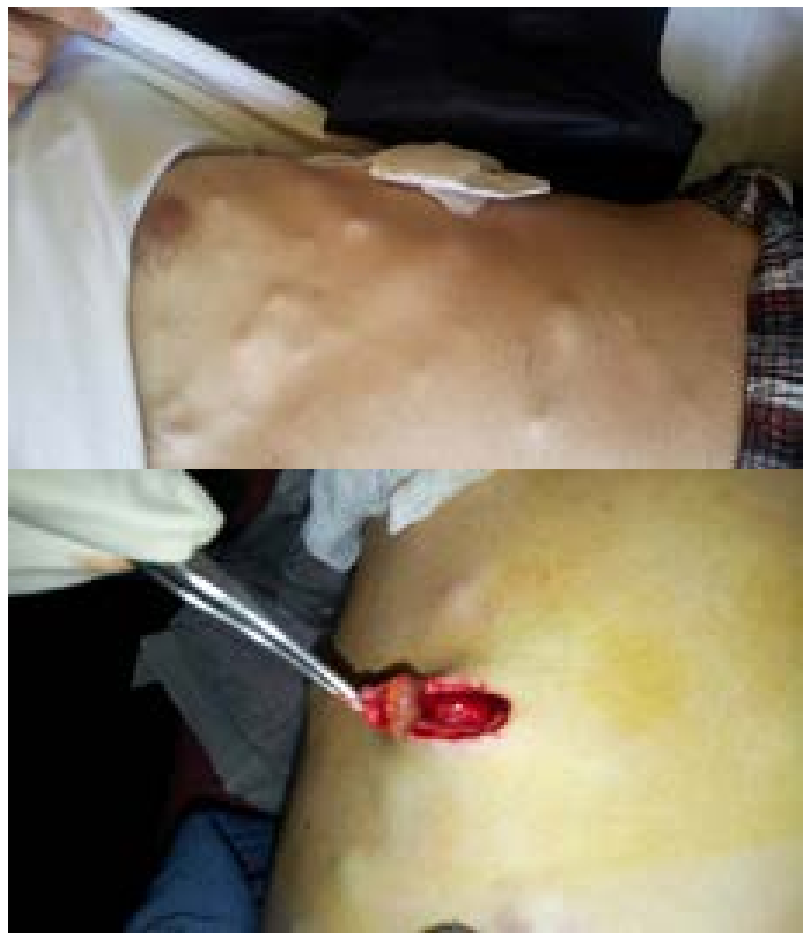

Figure 3 : Multiple skin metasis option is limited in our centre where the patient is referred to oncology department for chemotherapy.

\section{Conclusion}

As the malignant melanoma incidence is rising especially in young adults, increased general knowledge about skin cancers and protection from sun exposure in younger age group is important.

The early recognition of MM by physicians is lifesaving where surgery is definite treatment.

The periodic scientific activity for general physicians and residents to increase awareness about malignant melanoma is crucial to improve outcome

Lastly the hospital team work is essential for diagnosis of complicated cases. 